\title{
LOCALISED CHARGING EFFECTS INDUCED IN NON- CONDUCTIVE MATERIALS DURING FOCUSED ION BEAM MILLING
}

\author{
Marion A. Stevens-Kalceff,* Sergey Rubanov* and Paul R. Munroe** \\ * School of Physics and Electron Microscope Unit, University of New South \\ Wales, Sydney, NSW 2052, Australia. \\ **School of Materials Science and Engineering and Electron Microscope Unit, \\ University of New South Wales, Sydney, NSW 2052, Australia.
}

A Focused Ion Beam miller (FIB) produces a finely focused, energetic beam of gallium ions which may be scanned over the surface of a specimen. At reduced beam currents, the secondary electrons or secondary ions emitted from the specimen surface can be used to form high resolution images. At high beam currents the gallium beam sputters the specimen surface permitting accurately located subsurface cross-sections to be prepared. The FIB is particularly useful for the examination of materials where subsurface microstructure information is required. It is therefore important to assess the influence of ion implantation and ion milling on the specimen structure. Ion beam irradiation of poorly conducting materials may result in the trapping of charge at either pre-existing or implantation induced defects. [1] The trapped charge produces an electric field within the ionirradiated micro-volume of specimen which may influence the local structure. This work investigates focused ion beaminduced charging of insulating materials using advanced Scanning Probe Microscopy techniques.

Kelvin Probe Microscopy (KPM) or Scanning Surface Potential Microscopy (SSPM) is a specialized Atomic Force Microscopy technique in which long-range Coulomb forces between a conductive atomic force probe and a specimen enable the electrical potential at the specimen surface to be characterized with high spatial resolution. [2] A Veeco/ Digital Instruments extended Dimension 3000 Scanning Probe Microscope configured to operate in KPM/SSPM mode, has been used to characterize non-conductive materials exposed to gallium ion irradiation in a FEI $x P 200$ Focused Ion Beam miller.

Significant localized residual charging is observed within the gallium implanted micro-volumes of non-conductive materials prior to and following the onset of sputtering (see Fig. 1) Charge mitigation strategies including coating the specimen with a layer of thin grounded conductive material prior to milling and/ or the use of an electron flood gun during milling have been investigated. The degree of charging is influenced by a number of different dynamic processes including implantation, non-stoichiometric sputtering from compounds, secondary electron emission, secondary electron trapping by irradiation induced defects, etc.

The reproducible characteristic surface potentials associated with the trapped charge have been measured using Kelvin Probe Microscopy and modelled using three dimensional conformal Finite Element Analysis [3] and compared with observed residual potentials. This gives insight into the charging processes during implantation and sputtering and the resultant spatial distributions of the residual trapped charge. The results of this work have implications for the microanalysis of non-conductive materials processed in Focused Ion Beam syatems. 


\section{References}

[1] Lefebvre, F., Vigouroux, J. P. \& Perreau, J. Charge phenomena induced by low-energy ion bombardment $\mathrm{SiO}_{2}$. Journal of Applied Physics, 65(4), (1989). 1683-9.

[2] Bonnell, D. A. Scanning Probe Microscopy and Spectroscopy: Theory, Techniques and Applications. Wiley-VCH. New York, 2001.

[3] Field Precision, Albuquerque, NM

[4] Support from the Australian Research Council and the Electron Microscope Unit at University of New South Wales is gratefully acknowledged.

Marion.Stevens-Kalceff@unsw.edu.au

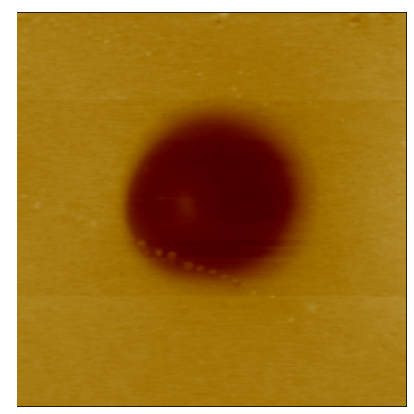

(a)

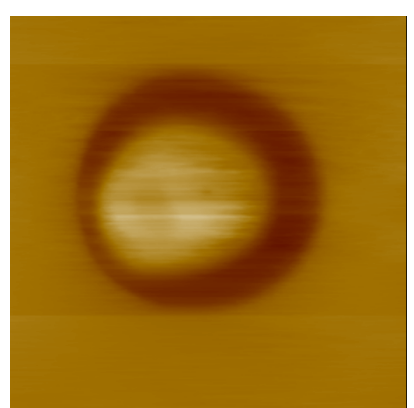

(b)

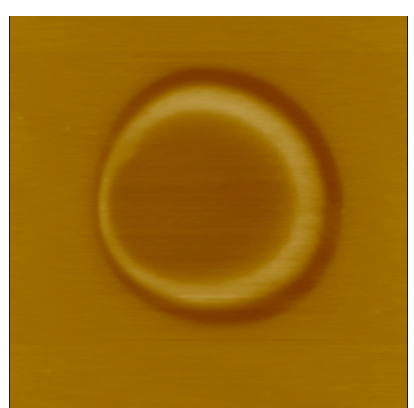

(c)

FIG. 1. 40x 40 $\mu \mathrm{m}^{2} \mathrm{KPM}$ images of $\mathrm{SiO}_{2}$ implanted/ sputtered in a Focused Ion Beam miller with a $30 \mathrm{keV}, 6600 \mathrm{pA}, 5 \mu \mathrm{m}$ diameter $\mathrm{Ga}+$ ion beam for (a) $0.5 \mathrm{~s}$. (b) $3 \mathrm{~s}$ and (c) 7.5s. The specimen was coated with a thin grounded layer of conductive material prior to implantation/sputtering. These images show significant residual surface potentials of several hundred $\mathrm{mV}$. 